\title{
Extinction effects in $x$-ray holographic imaging with internal reference
}

\author{
P. Korecki, ${ }^{1}$ D. V. Novikov, ${ }^{2}$ M. Tolkiehn, ${ }^{2}$ and G. Materlik ${ }^{3}$ \\ ${ }^{1}$ Institute of Physics, Jagiellonian University, Reymota 4, 30-059 Krakow, Poland \\ ${ }^{2}$ Hamburger Synchrotronstrahlungslabor HASYLAB am Deutschen Elektronen-Synchrotron DESY, 22603 Hamburg, Germany \\ ${ }^{3}$ Diamond Light Source Limited, Rutherford Appleton Laboratory, Chilton, Didcot, Oxfordshire OX11 OQX, United Kingdom
}

(Received 8 November 2003; published 20 May 2004)

\begin{abstract}
Secondary extinction effects in x-ray holography with internal atomic reference are examined in the frame of multiple-scattering power transfer equations for a mosaic crystal. The calculations are compared with experimental holograms of an imperfect $\mathrm{Cu}_{3} \mathrm{Au}$ single crystal measured using $\mathrm{x}$-ray fluorescence and total electron yields. It is shown that extinction effects in imperfect crystals can influence reconstructed real-space images in x-ray atom-resolving holography.
\end{abstract}

DOI: $10.1103 /$ PhysRevB.69.184103

PACS number(s): 61.10.-i, 42.40.- $\mathrm{i}, 61.18 .-\mathrm{j}$

\section{INTRODUCTION}

Internal x-ray source holography ${ }^{1}$ was suggested to solve the crystallographic phase problem. It uses atomic sources, emitting spherical x-ray waves, and employs a holographic formalism to analyze the pattern of the interference between the directly emitted internal reference wave and the waves scattered on neighboring atoms. Since these waves are added coherently, the measured intensity contains information about the relative phase of the object waves and thus a inversion of the hologram to the real space is feasible. ${ }^{2}$ This type of holography was performed for the first time with electrons ${ }^{3}$ and subsequently with $\mathrm{x}$ rays, ${ }^{4} \gamma$ rays, ${ }^{5}$ and neutrons. ${ }^{6}$ It is also performed in the time-reversed version, ${ }^{7}$ in which the interference of an external plane wave and scattered waves is monitored by absorption of particular atoms serving as internal detectors. The signal for probing the local $\mathrm{x}$-ray field intensity is provided by secondary yield and it is usually registered with an external detector. For a survey of x-ray holography, cf. review articles. ${ }^{8,9}$ The present paper discusses the time-reversed x-ray holography, but all of its conclusions may be extended to the direct version of $\mathrm{x}$-ray holography and to $\gamma$-ray and neutron holography.

Until now, all $\mathrm{x}$-ray holographic experiments were performed for single crystals. Simultaneously, in the present theories the holograms were usually calculated by summing the contributions from scatterers in small clusters (typically with radius $50 \AA$, containing maximal $10^{5}$ atoms) around the atoms of interest. The cluster approach ${ }^{10,11}$ originates from photoelectron holography in which all atomic sources are located near surface and the high absorption reduces the contributions from further scatterers. However, for $\mathrm{x}$ rays the presence of the scattering and absorbing medium outside such clusters can influence the holographic pattern. Because of the weak absorption of $x$ rays, the predominant features of $\mathrm{x}$-ray holograms recorded for both imperfect ${ }^{8,9}$ and perfect ${ }^{12,13}$ crystals are sharp lines resulting from long-range order. These lines are centered on reciprocal vectors and lie on the so-called Kossel cones corresponding to the Bragg condition. ${ }^{14}$ In experiments, the shape of these sharp features often differs from that predicted by the kinematical theory, ${ }^{15,16}$ and any angular dependent features that are not described by the kinematical model can cause a distortion of the real-space reconstruction of the holograms performed with existing algorithms. This deviation from the antisymmetrical shape was often attributed to the self-interference of the object waves but, as it will be shown, it is a oversimplified picture. Close to the Bragg angle, the kinematical theory is not valid. Extinction effects of the primary beam, caused by Bragg scattering, can be severe. ${ }^{17}$ Unlike in x-ray diffraction, where the extinction effects decrease the measured intensity, the extinction effects in $\mathrm{x}$-ray holography can have a more complex influence on the secondary yield. The secondary yield is excited by both the primary and scattered beams and, as it will be shown, can be decreased or increased at particular depth of the sample depending on the interaction between the incident and the scattered beams.

In a holographic experiments performed for imperfect crystals, the coherent interaction between beams (which is exploited in x-ray standing wave method ${ }^{18-21}$ ) is averaged, depending on crystal quality and/or experimental conditions. Therefore, the extinction theory of mosaic crystals (secondary extinction $)^{17,22}$ is suitable for application in x-ray holography. The secondary extinction model, adopted in the present work for $\mathrm{x}$-ray holography, uses the following scheme. Inside a single mosaic block the holographic picture can be used. The local reference wave (characteristic of a single mosaic block) interferes with object waves which are scattered inside the same crystallite, and this interference field is probed by an atomic detector. However, near the Bragg condition, the radiation is rescattered into other blocks. This radiation is then incoherently coupled with the incident beam in other mosaic blocks and can increase the secondary yield coming from a particular depth. Simultaneously, the incident beam is attenuated by both absorption and extinction. The extinction effects will thus strongly depend on the escape depth of the secondary signal. Figure 1 illustrates the extinction effects in in x-ray holography in the mosaic crystal model.

The paper is organized as follows. In Sec. II the absorption and extinction cross sections for a small spherical crystallite (single mosaic block) are calculated in the kinematical approximation. These cross sections are subsequently used as coupling constants of power transfer equation used for calculation of the secondary electron yield from a mosaic crystal. Section III compares the calculation with an experiment 


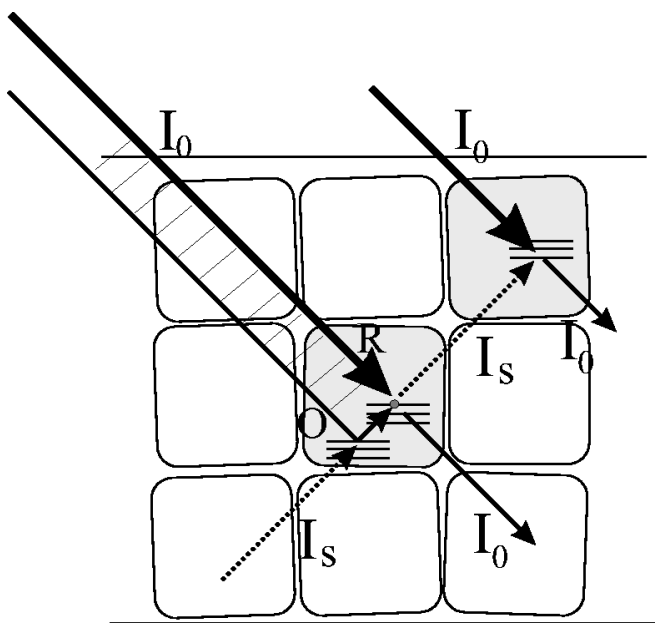

FIG. 1. Illustration of extinction effects in x-ray holography for a mosaic crystal. Inside a single mosaic block, a holographic picture of the interference between the reference $R$ and object waves $O$ can be used. However, the incident field $I_{0}$ at a given depth that gives rise to the local reference wave is already attenuated in blocks at lesser depth. Near the Bragg condition the radiation is additionally rescattered $\left(I_{s}\right)$, and it is incoherently coupled with the incident beam in other mosaic blocks. For details see text.

performed for an imperfect $\mathrm{Cu}_{3} \mathrm{Au}$ crystal. Section IV qualitatively discusses the influence of extinction effects on reconstructed real-space images.

\section{THEORY}

The calculation is performed under the following assumptions.

(i) The sample consists of spherical mosaic blocks, in which the absorption and scattering are small, i.e., $\mu R \ll 1$ and $\sigma R \ll 1$, where $R \gg a$ is the average size of a mosaic block and $a$ is the lattice constant. This means that the intensity of the radiation does not change significantly over the length comparable to $R$. For this reason, all the calculations correspond to the so-called secondary extinction approximation. If this condition is not fulfilled, so-called primary extinction may dominate. ${ }^{23}$

(ii) Inside a single mosaic block, the holographic modulation of the absorption cross section and the extinction cross section are calculated by coherent summation over all atoms inside the block.

(iii) Near the Bragg condition, radiation can be multiple scattered between blocks, and this changes the intensity of the total field inside the sample. The transfer of power between blocks is incoherent. The phase relation between the waves diffracted by two adjacent blocks is supposed to be random and this results in an incoherent process. The transfer between blocks is calculated using two-beam approximation.

\section{A. Absorption, extinction, and secondary yield of a single mosaic block}

Consider a plane wave with a wavelength of the order of $0.1 \mathrm{~nm}$, which irradiates a spherical crystallite with relatively small diameter $R$. The incident radiation is attenuated via absorption and scattering. For simplicity, it is assumed that only coherent scattering and photoelectric absorption contribute to the attenuation. Therefore, the total cross section for any attenuation process per unit volume of the crystallite may be written as

$$
\sigma_{t o t}(\vec{k})=\sigma(\vec{k})+\mu(\vec{k}),
$$

where $\sigma(\vec{k})$ is the extinction or the coherent scattering cross section per unit volume and $\mu(\vec{k})$ is the photoelectric absorption cross section per unit volume. $\vec{k}$ is the wave vector of the incident plane wave.

\section{B. Extinction}

The extinction cross section per unit volume of this spherical block can be calculated by an integral over the direction of the scattered beam $\vec{k}_{s}$ :

$$
\sigma(\vec{k})=\int \sigma\left(\vec{k}, \vec{k}_{s}\right) d \Omega_{k_{s}} .
$$

Generally, this calculation is not straightforward. For point scatterers and arbitrarily shaped objects, it can be calculated by a real-space summation. ${ }^{24}$ Since the real-space summation is extremely nonconvergent, ${ }^{25}$ such a calculation is limited to small $(5-10 \mathrm{~nm})$ clusters and cannot represent the approximate solution for bigger clusters.

For a crystalline spherical object containing nonpoint scatterers, the scattering cross section can be obtained near the Bragg condition corresponding to a reciprocal vector $\vec{H}:{ }^{26,27}$

$$
\sigma_{H}\left(\Delta \theta_{H}\right)=\frac{8 \pi^{2}}{k^{2}}\left|\frac{F_{H}}{V}\right|^{2} \frac{\Gamma}{\Gamma^{2}+\left(k \sin 2 \theta_{H} \Delta \theta_{H}\right)^{2}} .
$$

In Eq. (3) $\Delta \theta_{H}=\Delta \theta_{H}(\vec{k})$ is a small angular deviation of the incident beam from the exact Bragg condition measured in the scattering plane (the plane containing the incident and the diffracted beam as well as the reciprocal vector $\vec{H}$ ).

Equation (3) is a smoothed Lorentz version of the exact expression calculated for a sharp edged sphere,${ }^{27}$ and

$$
\Gamma=\frac{1}{\rho} \text {. }
$$

The parameter $\rho=\rho(\vec{H})$ is introduced here as the effective coherent size of the crystallite. Generally, it can also qualitatively describe the properties of the incident beam (energy spread and divergence). However, in this work it is assumed that $\rho=3 R / 4$, where $R$ is the radius of the crystallite. The Bragg angle corresponding to $\vec{H}$ is denoted as $\theta_{H}$. $F_{H}$ is the structure factor:

$$
F_{H}=\sum_{i=1}^{N} f_{i} e^{-i \vec{H} \cdot \vec{r}_{i}}
$$


and $f_{i}$ is the angular dependent atomic scattering factor of the $i$ th atom in the unit cell composed of $N$ atoms. $f_{i}$ contains the Debye-Waller and the polarization factor. For simplicity, the polarization factor is calculated for an unpolarized incident beam. $V$ is the volume of the unit cell.

The full two-dimensional directional dependence of $\sigma$ is calculated as a sum over reciprocal points:

$$
\sigma(\vec{k})=\sum_{H} \sigma_{H}\left(\Delta \theta_{H}\right)
$$

\section{Absorption}

Contrary to the extinction cross section for an intermediate size crystallite, which has to be evaluated only near Bragg conditions and otherwise is close to zero, the absorption cross section must be calculated over a full hemisphere. The average absorption cross section per unit volume may be divided into a constant part $\mu_{0}$, resulting from pure absorption, and an angular dependent part containing holographic oscillations: ${ }^{8}$

$$
\mu(\vec{k})=\mu_{0}+\mu_{s}(\vec{k}),
$$

where

$$
\mu_{0}=1 / V \sum_{j=1}^{N} \sigma_{j}^{a b s}
$$

and

$$
\mu_{s}(\vec{k})=1 / V \sum_{j=1}^{N} \sigma_{j}^{a b s} \chi_{j}(\vec{k})
$$

$\sigma_{j}^{a b s}$ is the absorption cross section of the $j$ th atom in the unit cell calculated for an isolated atom. The absorption cross section of an isolated atom $\sigma_{j}^{a b s}$ is calculated from tabulated values of the imaginary part of the atomic scattering amplitude $f_{j}^{\prime \prime}$ as $\sigma_{j}^{a b s} \approx-4 \pi / k f_{j}^{\prime \prime}$.

$\chi_{j}(\vec{k})$ is the hologram seen by the $j$ th atom in the central unit cell of the sphere. Since the crystallite has finite dimensions, all atoms, apart from the atoms related by symmetry, are nonequivalent. Therefore, the summation in Eq. (9) should be performed over all atoms in the crystallite and ought not to be restricted to atoms belonging to a single unit cell. However, because of the lack of the periodicity, the calculation of the exact expression is not readily performed. Only $N$ holograms characteristic of $N$ atoms in the central unit cell are calculated and they represent an effective hologram characteristic of $j$ th type of atoms. These holograms are calculated using a modified average shape of the crystallite, which to some extent compensates this approximation.

Holographic oscillations $\chi_{j}$ are calculated similarly as in previous works, i.e., as (see Refs. 15,16)

$$
\chi_{j}(\vec{k})=-2 r_{0} \operatorname{Re} \int d^{3} r \tilde{\rho}_{j}(\vec{r}) \frac{e^{i k\left|\vec{r}-\vec{r}_{j}\right|}}{\left|\vec{r}-\vec{r}_{j}\right|} e^{i \vec{k} \cdot\left(\vec{r}-\vec{r}_{j}\right)},
$$

where $\tilde{\rho}_{j}$ is the electron density of the crystallite with the $j$ th atom removed:

$$
\tilde{\rho}_{j}(\vec{r})=\rho(\vec{r})-\rho_{j}(\vec{r}),
$$

and $r_{0}$ is the classical electron radius.

$\tilde{\rho}_{j}$ is evaluated as a product of a reciprocal series of an infinite lattice $\rho_{\infty}$ and the shape function $S_{j}(\vec{r})$ :

$$
r_{0} \tilde{\rho}_{j}=r_{0} \tilde{\rho}_{\infty} S_{j}(\vec{r})=\left[1 / V \sum_{H} F_{H} e^{i \vec{H} \cdot \vec{r}}\right] S_{j}(\vec{r}) .
$$

In accordance with previous considerations, the shape function $S_{j}$ is different for each atom in the crystallite. To make the calculation simple, the effective hologram of the $j$ th atom in a unit cell is calculated only for the central unit cell of the sphere by using an effective spherically symmetric shape function $\widetilde{S}(\vec{r})$. Using this approximation, the angular dependent part of the absorption cross section may be written as

$$
\mu_{s}(\vec{k})=\frac{8 \pi}{V^{2}} \frac{1}{k} \operatorname{Re} \sum_{H} F_{H} G_{H}^{*} \int d^{3} r \widetilde{S}(r) e^{i(\vec{H}+\vec{k}) \cdot \vec{r}} \frac{e^{i k r}}{r} .
$$

The factor $G_{H}$,

$$
G_{H}=\sum_{j=1}^{N} f_{j}^{\prime \prime} e^{-i \vec{H} \cdot \vec{r}_{j}}
$$

is similar to the structure factor $F_{H}$, but it contains only the imaginary parts of the atomic scattering amplitudes $f_{j}^{\prime \prime}$, which also include the Debye-Waller factor. Of course the effective shape function $\widetilde{S}(r)$ cannot be chosen ad hoc. The form of the shape function can be derived from the realspace calculations of extinction and absorption for small clusters containing point scatterers. The extinction and absorption patterns calculated for pure imaginary scattering amplitudes and for centrosymmetric systems have, apart from amplitude, the same form. ${ }^{24,28}$ Thus, in order to calculate the absorption pattern which is consistent with the calculation of extinction, $\widetilde{S}(r)$ has to equal as follows:

$$
\widetilde{S}(r)=e^{-\Gamma r} .
$$

This form of $\widetilde{S}(r)$ qualitatively takes into account the finite dimension of the spherical crystallite. It mimics the autocorrelation function of the sphere with radius $R: s(r)$ $=(R-r / 2)^{2}(2 R+r / 2) /\left(2 R^{3}\right)$.

For this form of $\widetilde{S}(r)$ the angular dependent part of the absorption cross section becomes

$$
\mu_{s}(\vec{k})=\left|\frac{4 \pi}{V}\right|^{2} \frac{1}{k} \operatorname{Re} \sum_{H} F_{H} G_{H}^{*} \frac{1}{q} \Phi_{\mu}(\vec{k}),
$$

where

$$
\Phi_{\mu}(\vec{k})=€\left(\vec{k}_{+}\right)-\succeq\left(\vec{k}_{-}\right)
$$




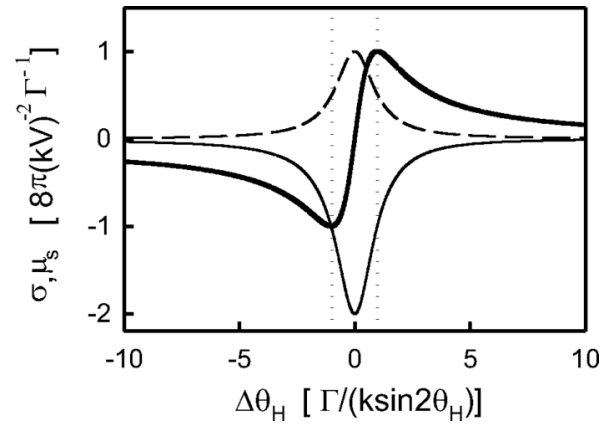

FIG. 2. Directional dependence of absorption $\mu_{s}$ and extinction $\sigma$ cross sections in a single mosaic block plotted in the function of the angular deviation $\Delta \theta_{H}$ from the Bragg angle in the scattering plane. Extinction (dashed line) is calculated for $\left|F_{H}\right|^{2}=1$. The real (thick solid line) and the imaginary (thin solid line) parts of absorption for $G_{H}=1$ and for $F_{H}=1$ or $F_{H}=i$, respectively. The vertical dotted lines correspond to FWHM of the symmetrical curves.

and

$$
E(x)=\frac{x-i \Gamma}{\Gamma^{2}+x^{2}}
$$

The auxiliary variables introduced in Eq. (16) are

$$
q=|\vec{H}+\vec{k}|, k_{+}=k+q, k_{-}=k-q .
$$

Equation (16), apart from $G_{H}$, is similar to the expression in other works. ${ }^{15,16}$

It is noteworthy that by a suitable approximation one can show that $\mu_{s}$ has very similar $\sin 2 \theta_{B}$-like dependence on the angular deviation from the Bragg condition to the expression for extinction.

Figure 2 shows the directional dependence of absorption $\mu_{s}$ and extinction $\sigma$ cross sections plotted in the function of the angular deviation $\Delta \theta_{H}$ from the Bragg angle in the scattering plane calculated from Eqs. (3) and (16), respectively.

All curves have the same widths. The ratio of theirs amplitudes is only determined by $F_{H}$ and $G_{H}$. The real part of the absorption cross section has an almost antisymmetric shape, whereas the imaginary parts of the absorption cross section and the extinction cross section have an almost symmetrical shape.

\section{Secondary yield}

For the calculation of secondary yield from the absorbing crystalline, it is worth introducing

$$
\mu^{\text {det }}(\vec{k})=\mu_{0}^{d e t}+\mu_{s}^{\text {det }}(\vec{k}),
$$

i.e., the absorption cross section per unit volume of the atoms emitting secondary yield. It is calculated in the same manner as $\mu$ with $G_{H}$ replaced by

$$
D_{H}=\sum_{i=1}^{M} f_{i}^{\prime \prime} e^{-i \vec{H} \cdot \vec{r}_{i}}
$$

In Eq. (21), the summation is over $M$ atoms in the unit cell giving rise to secondary yield, and hereafter referred to as detecting atoms.

\section{E. Mosaic spread}

In the subsequent section, $\mu, \mu^{d e t}$, and $\sigma$ are calculated to represent the average cross sections per unit volume in a mosaic crystal. It is assumed that the angular distribution of mosaic blocks is independent of the position in the sample. The coefficients $\sigma, \mu$, and $\mu^{\text {det }}$ are than replaced by the convolutions

$$
\sigma \rightarrow \sigma^{*} W, \quad \mu \rightarrow \mu^{*} W, \quad \mu^{d e t} \rightarrow \mu^{\operatorname{det} *} W,
$$

where $W$ is the mosaic spread function.

In the calculation it is performed by replacing

$$
\Gamma \rightarrow \Gamma+\frac{k \sin 2 \theta_{H} \Delta W}{2},
$$

where $\Delta W$ is the full width at the half maximum (FWHM) of the mosaic distribution. This approximately corresponds to a convolution with a Lorentzian mosaic distribution.

\section{F. Secondary yield calculated for a mosaic crystal}

If the direction of the incident beam is close to the Bragg condition, a revised formalism is needed to calculate the hologram. Such a suitable and self-consisted formalism based on the Darwin multiple-scattering equation ${ }^{22,29}$ was recently described by Sears ${ }^{30}$ and it is used in this section to calculate the secondary yield.

The calculation of the directional dependence of the secondary yield is performed for a crystalline sample in the form of a slab with infinite lateral extent and with the entrance surface at $z=0$ and the exit surface at $z=d$. For such a system the total number of photons inside the sample at depth $z$, i.e., $I(z)$ [in the text of the paper, $I(z)$ represents the total power in the beam in photon/s or the neutron current in neutron/s] depends only on the depth inside a sample.

For $0<z<d$, the equations describing the power transfer between the primary beam $I_{0}(z)=I_{0}(z, \vec{k})$ and the scattered beam $I_{s}(z)=I_{s}(z, \vec{k})$ are

$$
\begin{aligned}
& \frac{d I_{0}(z)}{d z}=-\tau_{00} I_{0}(z)+\tau_{s 0} I_{s}(z), \\
& \pm \frac{d I_{s}(z)}{d z}=-\tau_{s s} I_{s}(z)+\tau_{0 s} I_{0}(z),
\end{aligned}
$$

where $[+]$ is for the Laue forward-scattering geometry (scattered beam leaves through exit surface) and $[-]$ for the Bragg backscattering geometry (scattered beam leaves through entrance surface).

The coupling constants are

$$
\tau_{00}=\frac{\sigma(\vec{k})+\mu(\vec{k})}{\sin \theta_{0}}, \quad \tau_{s 0}=\frac{\sigma\left(\overrightarrow{k_{s}}\right)}{\sin \theta_{s}},
$$




$$
\tau_{s s}=\frac{\sigma\left(\overrightarrow{k_{s}}\right)+\mu\left(\overrightarrow{k_{s}}\right)}{\sin \theta_{s}}, \quad \tau_{0 s}=\frac{\sigma(\vec{k})}{\sin \theta_{0}} .
$$

$\theta_{s}$ and $\vec{k}_{s}$ are the positive glancing angle and the wave vector of the diffracted beam.

For the Laue forward-scattering geometry, the boundary conditions are

$$
I_{0}(0)=1, I_{s}(0)=0,
$$

and for the Bragg back-scattering geometry

$$
I_{0}(0)=1, I_{s}(d)=0 .
$$

Equation (24) can be solved analytically and the closed form of the solution can be obtained. ${ }^{30}$

The secondary yield $Y$ (particle/s) of atoms of interest can be calculated from

$$
Y(\vec{k})=\int_{0}^{d} P(z)\left[y_{0}(z)+y_{s}(z)\right] d z,
$$

where

$$
y_{0}(z)=\frac{\mu^{d e t}(\vec{k})}{\sin \theta_{0}} I_{0}(z), \quad y_{s}(z)=\frac{\mu^{d e t}\left(\overrightarrow{k_{s}}\right)}{\sin \theta_{s}} I_{s}(z)
$$

are the absorption per unit depth of the primary and scattered beams, respectively. $P(z)$ is the probability that the secondary particle emitted at depth $z$ reaches the detector. For simplicity, it is assumed that

$$
P(z)=\exp \left(-\mu^{s e c} z\right),
$$

where $1 / \mu^{s e c}$ is the escape depth of the secondary radiation.

If $\mu^{d e t}=\mu$, the secondary yield can also be calculated from the total power loss at depth $z$ :

$$
Y(\vec{k}) \propto-\int_{0}^{d} P(z)\left(\frac{d I_{0}(z)}{d z} \pm \frac{d I_{s}(z)}{d z}\right) d z,
$$

where \pm corresponds to the Laue and the Bragg geometry, respectively.

For $P(z)=1$, the detected secondary yield $Y$ is equal to the total absorption $A$ of the sample.

From Eqs. (24)-(28), it follows that

$$
R+T+A=1,
$$

where $T=I_{0}(d)$ is the transitivity of the sample and $R$ is the reflectivity of the sample; for the Laue geometry $R$ $=I_{H}(d)$, while for the Bragg geometry $R=I_{H}(0)$.

Equation (32) gives the conservation of energy (particles) in the system. In addition Eq. (32) is used as a reference check for calculations.

\section{COMPARISON WITH EXPERIMENT}

In order to examine the extinction effects, $\mathrm{x}$-ray holograms were recorded for an imperfect $\mathrm{Cu}_{3} \mathrm{Au}$ single crystal using secondary yield signals having significantly different escape depths: X-ray fluorescence yield (XFY) and total electron yield (TEY). The sample used in the experiment was a polished $\mathrm{Cu}_{3} \mathrm{Au}$ crystal in the ordered phase with the surface parallel to (001) direction. The mosaicity of the sample was $\sim 0.2^{\circ}$. Holographic experiments had been already performed on such samples. ${ }^{15,31}$ The measurements were carried out with synchrotron radiation at HASYLAB on beamline CEMO using a $\mathrm{Ge}$ (111) double-crystal monochromator at energy $10 \mathrm{keV}$. The $\mathrm{Cu} K \alpha, \beta$ x-ray fluorescence was measured using a unbiased PN photodiode (active area diameter $20 \mathrm{~mm}$ ) similar as in Ref. 32. The average measured current was $\sim 0.1 \times 10^{-10} \mathrm{~A}$. The diode was tilted with respect to the sample surface normal by $40^{\circ}$ and placed $80 \mathrm{~mm}$ from the sample. For this observation angle the effective escape depth of the x-ray $\mathrm{Cu} K$ fluorescence is $\sim 5 \mu \mathrm{m}$. In addition, $25 \mu \mathrm{m} \mathrm{Ni}$ and $\mathrm{Zn}$ absorber foils were placed in front of the diode in order to suppress the elastically scattered radiation. To monitor for a possible contamination of the signal by Bragg-reflected primary beam, the whole pattern was simultaneously measured with a silicon drift detector. The total electron yield was collected by placing the sample in a $\mathrm{He}$ filled chamber and biasing it to $-90 \mathrm{~V}$. The average measured current was $\sim 0.5 \times 10^{-9} \mathrm{~A}$. The value of the escape depth of the electron signal was estimated to be $\sim 0.2 \mu \mathrm{m} .{ }^{33,34}$ In order to exclude any signal coming from the reflection of the primary beam into $\mathrm{He}$, parts of the pattern were measured with the sample placed in a vacuum chamber with $p \sim 5 \times 10^{-7}$ Torr in a separate experiment. Both XFY and TEY were measured simultaneously as a function of the sample orientation relative to the beam direction. The slow scan was performed by changing the azimuthal angle $\Phi$ with a step of $\Delta \Phi=0.5^{\circ}$ and in the range $\left(0^{\circ}, 180^{\circ}\right)$. For time limitation, some part of the patterns was measured on a $\Delta \Phi=1^{\circ}$ grid. The polar angle $\Theta$ was changed continuously and integrated over $\Delta \Theta \sim 0.1^{\circ}$ for $\sim 1 \mathrm{~s}$. The maximum polar angle was limited by the entrance window of the chamber to approximately $56^{\circ}$. The measured patterns were normalized using a signal from an ionization chamber. The background was removed separately for each $\Phi$ scan by a polynomial fit. The missing area for $\Phi$ $=\left(180^{\circ}, 360^{\circ}\right)$ was obtained using the twofold symmetry of the pattern. No other processing was applied to the patterns.

Figures 3(a) and 3(b) show the measured XFY and TEY patterns in the orthographic projection. Figure 3(c) shows the positions of the predominant Kossel-Bragg lines. Some sparse contamination (four short white lines) from Bragg reflection can be seen for the XFY pattern. Although XFY and TEY holograms have the same symmetry and similar slowly varying features, the profiles of the sharp lines corresponding to the Bragg conditions are completely different. These differences cannot be attributed neither to different atoms serving as internal detectors (the XFY comes exclusively from the $\mathrm{Cu}$ atoms, while the TEY has also a contribution from $\mathrm{Au}$ atoms) nor to different mosaic block distribution near the surface of the sample and in the bulk. Almost all differences between TEY and XFY are caused by extinction. According to the kinematical results of Sec. II A, the secondary yield for a centrosymmetrical sample should show a antisymmetrical black-white contrast (deficit/excess in the secondary yield) across the Kossel cone. Such a behavior can be ob- 

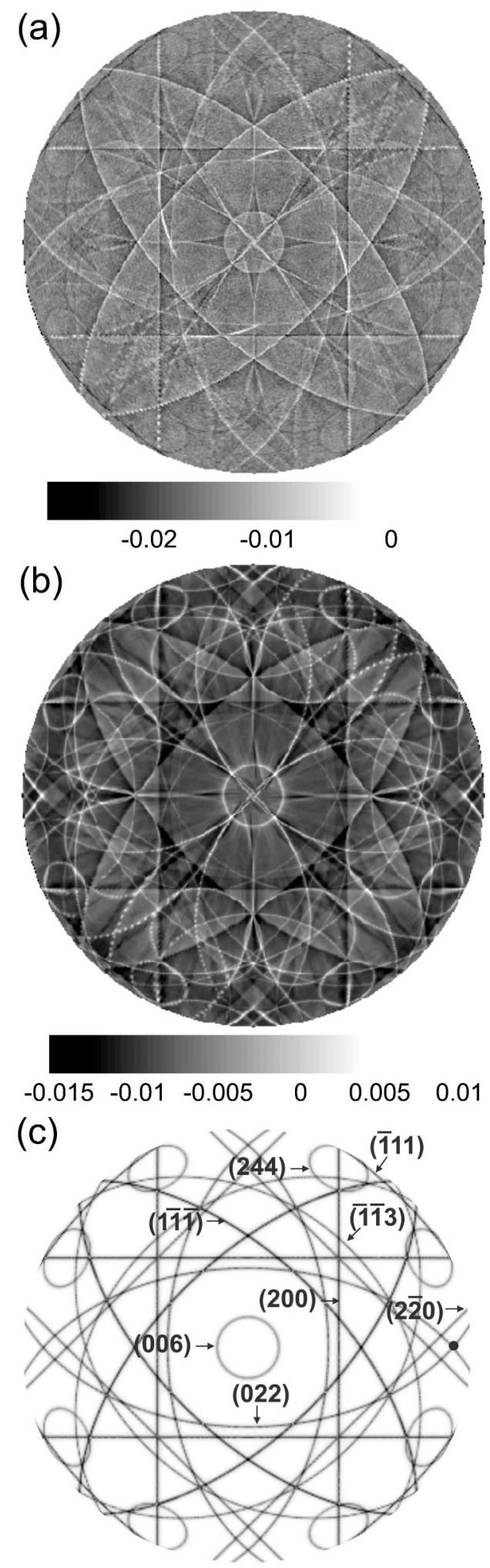

FIG. 3. Extinction effects in experimental x-ray holograms of a $\mathrm{Cu}_{3} \mathrm{Au}$ at $10 \mathrm{keV}$. (a) X-ray hologram measured using $\mathrm{Cu} K$ fluorescence secondary signal (XFY). (b) X-ray hologram measured using total electron secondary signal (TEY). (c) Positions of the Kossel lines on the hemisphere. The black circle denotes the transition from Bragg to Laue geometry for the (022) line.

served for the (200) line, for which extinction does not influence the secondary yield at all, for both XFY and TEY. The (200) line corresponds to a unique symmetrical reflection in the Laue geometry (forward-scattered beam goes into the sample). In contrast, for the (006) line in the symmetrical Bragg geometry (backscattered beam leaves through the entrance surface) the extinction effects influence both TEY and XFY. For TEY, this line can be observed as a white line (excess in the integrated intensity), while for XFY it shows a small deficit in the integrated intensity. In the TEY pattern the (244) and (11) 3 ) lines (Bragg geometry) are strongly enhanced, while the lines corresponding to the Laue geometry, $(1 \overline{1} \overline{1}),(\overline{1} 11)$ are hardly influenced by extinction. Furthermore, the rapid drop in the intensity corresponding to the Bragg-Laue transition is especially apparent for the (022) line in the TEY signal. The (111) line has a decreased intensity, while the otherwise symmetrically equivalent (11̄i) line has an increased intensity in the XFY pattern. It results from different asymmetry parameters $\xi=\sin \theta / \sin \theta_{s}$ of these reflections.

Figures 4(a) and 4(b) show patterns simulated for XFY an TEY with parameters corresponding to the experiment. The calculation was based on power transfer equations as described in Sec. II F For comparison, Fig. 4(c) shows the hologram calculated without extinction effects for a single mosaic block. All patterns were calculated for $\mathrm{Cu}$ reference atoms, an average mosaic block diameter of $0.1 \mu \mathrm{m}$, and mosaic angular spread $0.2^{\circ}$. The agreement between experimental patterns and calculation is very good for both XFY and TEY. The main differences result from noise, imperfect background subtraction, and approximations used in the calculation. All extinction affected lines corresponding to the Bragg condition are properly described by the theory. Simultaneously, the calculation predicts that, independent of the secondary yield, the lines in the symmetric Laue geometry are not affected by extinction and that for the asymmetric Laue, extinction is important only in XFY pattern.

\section{DISCUSSION. INFLUENCE OF EXTINCTION EFFECTS ON RECONSTRUCTED REAL-SPACE IMAGES}

The experiment and calculation show that the secondary yield emitted from an imperfect crystal is strongly influenced by extinction effects. The most important question is if the reconstructed real-space images are also influenced by these effects. Extinction produces sharp symmetric lines lying on the Kossel cones. For simplicity, let us assume that such features, corresponding to a pair of lattice vectors $\vec{H}$ and $-\vec{H}$, can be approximated using Dirac $\delta$ distributions:

$$
\chi(\theta, \phi)=c_{+} \delta\left(\theta-\theta_{H}\right)+c_{-} \delta\left(\theta-\theta_{-H}\right),
$$

where $\theta$ and $\phi$ are the polar and azimuthal angles in the coordinate frame with $z$ axis parallel to $\vec{H}, \theta_{H}=\pi / 2-\theta_{B}$ and $\theta_{-H}=\pi / 2+\theta_{B}, \theta_{B}$ being the Bragg angle. The factors $c_{+}$ and $c_{-}$are the amplitudes of the lines for $\vec{H}$ and $-\vec{H}$, respectively. The spherical integral transform ${ }^{2}$ to the real space, 

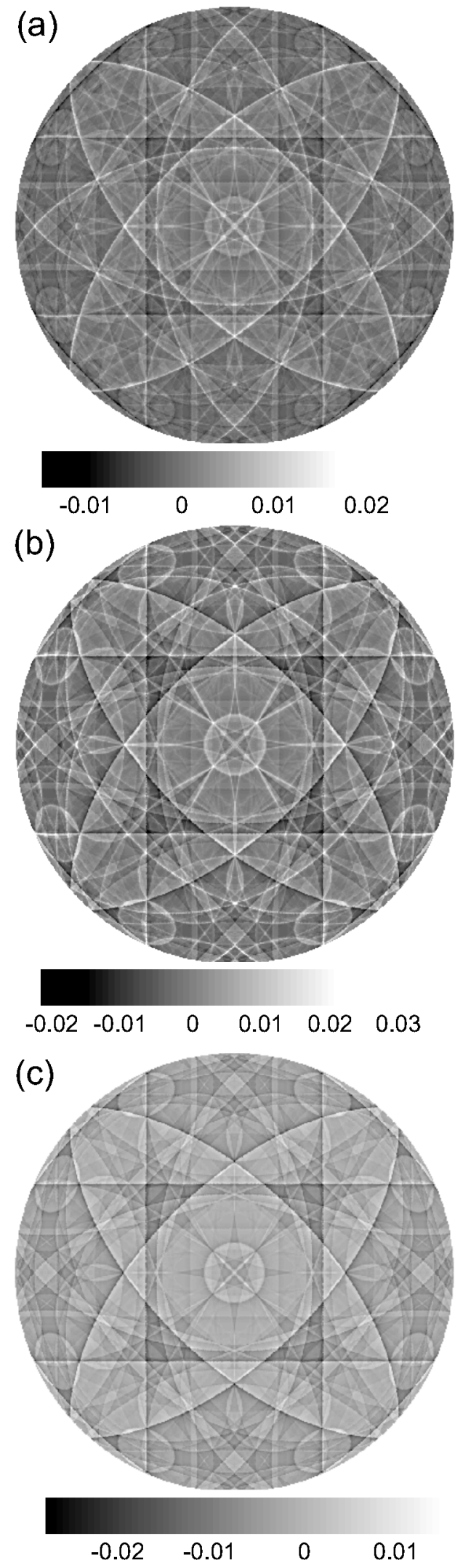

FIG. 4. Extinction effects in calculated x-ray holograms of a $\mathrm{Cu}_{3} \mathrm{Au}$ at $10 \mathrm{keV}$. (a) Calculated $\mathrm{x}$-ray hologram corresponding to XFY. (b) Calculated x-ray hologram corresponding to TEY. (c) $\mathrm{X}$-ray hologram calculated without extinction. All patterns are shown as orthographic projections. (a)
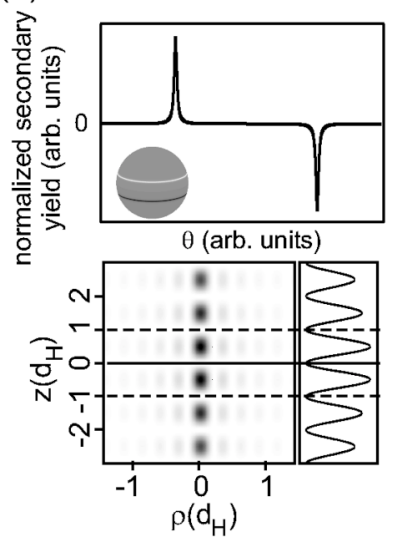

(b)

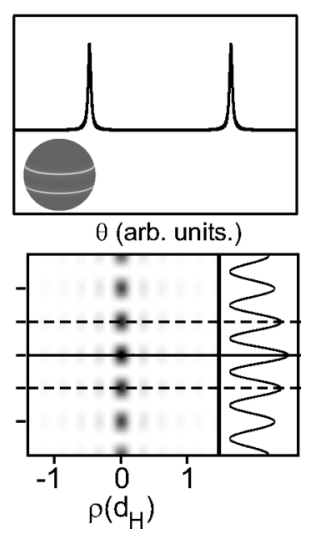

FIG. 5. Extinction effects in real-space images. Top: Angular profiles of the extinction lines corresponding to a pair of reciprocal vectors $\vec{H}$ and $-\vec{H}$. Insets show the positions of the lines on the sphere. Bottom: reconstructed cut of the real space. (a) and (b) correspond to two different cases described in the text.

$$
U(\vec{r})=\int_{S_{k}} \chi(\vec{k}) e^{i \vec{k} \cdot \vec{r}} d \Omega_{k}
$$

then gives

$$
\begin{aligned}
U(\rho, z)= & 2 \pi k^{2} \cos \left(\theta_{B}\right) J_{0}\left(k \rho \cos \theta_{B}\right)\left[\left(c_{+}+c_{-}\right) \cos \left(\pi \frac{z}{d_{H}}\right)\right. \\
& \left.+i\left(c_{+}-c_{-}\right) \sin \left(\pi \frac{z}{d_{H}}\right)\right],
\end{aligned}
$$

where $\rho$ and $z$ are cylindrical coordinates in the same coordinate frame and $d_{H}$ is the lattice plane spacing. Figure 5 shows extinction lines, for a pair of symmetrical reciprocalspace vectors, calculated with the same formalism as the patterns in Figs. 4(a) and 4(b) and the corresponding numerical real-space reconstruction $|U(\rho, z)|^{2}$. Figure 5(a) roughly corresponds to situation of $(\overline{1} 11)$ and $(1 \overline{1} \overline{1})$ reflections when $c_{+} \approx-c_{-}$, while Fig. 5(b) to the situation $c_{+} \approx c_{-}$. The latter case can appear as an artifact if a $4 \pi$ symmetrization $^{35,36}$ is performed on the experimental pattern recorded only for a hemisphere. Equation (35) and Fig. 5 show that extinction produces artifact images which have periodicity of a particular lattice plane spacing and a phase shift depending on the ratio between $c_{+}$and $c_{-}$and lie on the direction of $\vec{H}$. Recently it was shown that such effects cannot be fully removed by Fourier filtering of the holograms. ${ }^{37}$

The shape (intensities and widths) of extinction lines depends on the mosaic spread, the detection geometry, the energy of the incident radiation, and the absorption and the scattering cross sections, and has to be calculated from power-transfer equation [Eq. (24)] for a given crystal and an experimental geometry. However, for an arbitrary crystal and an arbitrary experimental geometry, one can estimate the amplitude of artefacts induced by extinction in the real space. Let us assume that the extinction lines visible in the hologram have Lorenztian shape: $L(\theta)=\pi^{-1} \gamma /\left(\gamma^{2}+\theta^{2}\right)$, where 
$\gamma$ is the angular width of the observed lines, which is determined by the mosaicity (mean radius and angular spread of mosaic blocks), incident radiation energy, and the finite angular resolution. Replacing $\delta(\theta)$ by $L(\theta)$ in Eq. (33) and inserting it into Eq. (34) one can show that the extinctioninduced features at distance $z$ (for $\rho=0$ ) have maximum amplitude $U_{e}(z) \approx(2 \pi k)^{2} A \gamma \cos \left(\theta_{B}\right) \exp (-k z \gamma)$. The measurable intensity $A$ of the extinction lines (maximum value normalized to the constant background) is connected with $c_{ \pm}$ of Eq. (33) by $A=c /(\pi \gamma)$, where for simplicity it is assumed that $c=c_{+}=c_{-}$. For comparison, a single point scatterer, located at distance $z$ from the detector atom, produces a hologram $\chi(\theta)=2 \bar{f} \cos (k z-k z \cos \theta) / z$, where $\theta$ is measured relative to the line joining both atoms, and $\bar{f}$ $=(4 \pi)^{-1} \int f(\theta) d \Omega$ is an effective real-valued scattering amplitude. The reconstruction of this hologram to the real space, using Eq. (34), produces two peaks in the real space (the real and the twin image) which have the amplitude $U_{1}(z)=4 \pi k^{2} \bar{f} / z$. For example, taking some realistic values, $\bar{f}=15 r_{0}, \quad E=10 \mathrm{keV}, \quad z=a_{0}=3.96 \AA, \quad \gamma=0.1^{\circ}, \quad A=0.02$ (similar as in holograms measured for $\mathrm{Cu}_{3} \mathrm{Au}$ ), and (220) reflection in a cubic lattice one gets $U_{1} / U_{e} \approx 1$.

Therefore, extinction, in addition to the twin images ${ }^{11}$ and limited k-space range, can lead to artifacts in the real-space reconstruction performed for imperfect crystals. Since the spurious images can occupy the correct atomic positions, they could lead to a false conclusions about the quality of the real-space reconstructions in previous experiments.

It is likely that the extinction-induced artefacts can be suppressed by multiple energy algorithms. ${ }^{38,39}$ In such algorithms, reconstructions corresponding to different energies (wave-vector values) are multiplied by a phase factor and coherently added, which can be approximately written as

$$
U_{M}(\rho, z)=\int_{k_{\min }}^{k_{\max }} k^{-2} U(\rho, z) e^{i k r} d k,
$$

where $U_{M}$ is the new real-space image. Since the phase of the reconstructed real image of $j$ th atom behaves like $e^{-i k r_{j}}$, such images are enhanced by this multienergy algorithm. Twin images (having phase $e^{i k r_{j}}$ ) and spurious images with different phase dependence are strongly suppressed. ${ }^{11,38}$ From Eq. (35) it follows that the extinction induced artifacts have different phase behavior than the exponent from Eq. (36), and this indicates that they could be removed in the same way as twin images. However, this possibility has to be verified in numerical stimulations.

The recently proposed single-energy algorithms, ${ }^{16,40,41}$ will be also influenced by extinction. Furthermore, it must be checked if diverse algorithms ${ }^{13,42,43}$ for twin-image removal are feasible to suppress the extinction effects. It is noteworthy that similar problems arise even for powder samples in the photon interference $\mathrm{x}$-ray absorption fine structure $(\pi$ XAFS $)$ method, ${ }^{44-46}$ which is an angle-integrated version of holography, and in recently proposed x-ray-absorption holography ${ }^{47}$ and angular integrated $x$-ray elastic-scattering methods. ${ }^{24}$ The reader is referred to a discussion concerning these papers. ${ }^{48,49}$

\section{CONCLUSIONS}

It was shown that extinction effects in large imperfect crystals having an approximate mosaic structure can influence both x-ray holograms and real-space reconstruction. Contrary to the X-ray standing wave (XSW) method where extinction is important only for XFY detection (i.e., when probing depth of the secondary radiation is grater than socalled extinction length), extinction effects in an holographic experiment performed on an imperfect sample are also important for TEY i.e. for short probing depths. The role of extinction effects requires more extensive studies. In particular similar effects should be examined in the direct version of $\mathrm{x}$-ray holography and for $\gamma$-ray and neutron holography. These effects may be reduced by an optimization of experimental strategy. For example, use of high energy or broadband illumination ${ }^{50,51}$ will strongly reduce the extinction effects. In these cases almost all the scattering take place close to symmetrical Laue geometry and thus it will be hardly affected by extinction. Another possibility is to use thin or small samples or samples with very small average diameter of mosaic blocks.

The simple theory, which is based on power-transfer equation, was verified by a comparison with experiment and can be used for more quantitative description of the extinction effects in the reconstructed real-space images for crystals having an approximate mosaic structure. Based on the presented theory the existing reconstruction algorithm can be modified in order to take into account the extinction effects. However, for other sorts of imperfect crystals (bent, strained crystal) more adequate theories ${ }^{20}$ should be used for quantitative analysis of the extinction effects.

\section{ACKNOWLEDGMENTS}

This work was supported by Volkswagenstiftung, Federal Republic of Germany (Project No. I/77 862) and by IHP Contract No. HPRI-CT-1999-00040/2001-00140 of the European Commission. P.K. would like to acknowledge the guidance and inspiration from Professor M. Szymoński.

\footnotetext{
${ }^{1}$ A. Szöke, in Short Wavelength Coherent Radiation: Generation and Applications, edited by D. T. Attwood and J. Bokor, AIP Conf. Proc. No. 147 (AIP, New York, 1986).

${ }^{2}$ J.J. Barton, Phys. Rev. Lett. 61, 1356 (1988).

${ }^{3}$ G.R. Harp, D.K. Saldin, and B.P. Tonner, Phys. Rev. Lett. 65,
}

1012 (1990).

${ }^{4}$ M. Tegze, G. Faigel, Nature (London) 380, 49 (1996).

${ }^{5}$ P. Korecki, J. Korecki, and T. Ślezak, Phys. Rev. Lett. 79, 3518 (1997).

${ }^{6}$ L. Cser, B. Farago, G. Krexner, I. Sharkov, and G. Torok, Phys. 
Rev. Lett. 89, 175504 (2002).

${ }^{7}$ T. Gog, P.M. Len, G. Materlik, D. Bahr, C.S. Fadley, and C. Sanchez-Hanke, Phys. Rev. Lett. 76, 3132 (1996).

${ }^{8}$ G. Faigel and M. Tegze, Rep. Prog. Phys. 62, 355 (1999).

${ }^{9}$ G. Faigel and M. Tegze, Struct. Chem. 14, 15 (2003).

${ }^{10}$ S. Thevuthasan, R.X. Ynzunza, E.D. Tober, C.S. Fadley, A.P. Kaduwela, and M.A. Van Hove, Phys. Rev. Lett. 70, 595 (1993).

${ }^{11}$ P.M. Len, S. Thevuthasan, C.S. Fadley, A.P. Kaduwela, and M.A. Van Hove, Phys. Rev. B 50, 11275 (1994).

${ }^{12}$ K. Hayashi, M. Matsui, Y. Awakura, T. Kaneyoshi, H. Tanida, and M. Ishii, Phys. Rev. B 63, 041201(R) (2001).

${ }^{13}$ Y. Nishino, T. Ishikawa, K. Hayashi, Y. Takahashi, and E. Matsubara, Phys. Rev. B 66, 092105 (2002).

${ }^{14}$ T. Gog, D. Bahr, and G. Materlik, Phys. Rev. B 51, 6761 (1995).

${ }^{15}$ B. Adams, D.V. Novikov, T. Hiort, G. Materlik, and E. Kossel, Phys. Rev. B 57, 7526 (1998).

${ }^{16}$ S. Marchesini, N. Mannella, C.S. Fadley, M.A. Van Hove, J.J. Bucher, D.K. Shuh, L. Fabris, M.J. Press, M.W. West, W.C. Stolte, and Z. Hussain, Phys. Rev. B 66, 094111 (2002).

${ }^{17} \mathrm{R}$. W. James, The Optical Principles of the Diffraction of X-Rays (Ox Bow Press, Woodridge, CT, 1962).

${ }^{18}$ J.A. Golovchenko, B.W. Batterman, and W.L. Brown, Phys. Rev. B 10, 4239 (1974).

${ }^{19}$ M.J. Bedzyk and G. Materlik, Phys. Rev. B 32, 6456 (1985).

${ }^{20}$ I.A. Vartanyants and M.V. Kovalchuk, Rep. Prog. Phys. 64, 1009 (2001).

${ }^{21}$ J. Zegenhagen, Surf. Sci. Rep. 18, 199 (1993).

${ }^{22}$ G.C. Darwin, Philos. Mag. 43, 800 (1922).

${ }^{23}$ P. Becker, Acta Crystallogr., Sect. A: Cryst. Phys., Diffr., Theor. Gen. Crystallogr. 33, 243 (1977).

${ }^{24}$ G. Faigel, M. Tegze, G. Bortel, and L. Koszegi, Europhys. Lett. 61, 201 (2003).

${ }^{25}$ P.P. Ewald, Ann. Phys. 64, 253 (1921).

${ }^{26}$ P.J. Becker and P. Coppens, Acta Crystallogr., Sect. A: Cryst. Phys., Diffr., Theor. Gen. Crystallogr. 30, 129 (1974).

${ }^{27}$ N.C. Popa, Acta Crystallogr., Sect. A: Found. Crystallogr. 43, 304 (1987).

${ }^{28}$ P. Korecki, J. Korecki, and W. Karas, Phys. Rev. B 59, 6139 (1999).

${ }^{29}$ W.C. Hamilton, Acta Crystallogr. 10, 629 (1957).
${ }^{30}$ V.F. Sears, Acta Crystallogr., Sect. A: Found. Crystallogr. 53, 35 (1997).

${ }^{31}$ D.V. Novikov, B. Adams, T. Hiort, E. Kossel, G. Materlik, R. Menk, and A. Walenta, J. Synchrotron Radiat. 5, 315 (1998).

${ }^{32}$ E. Busetto, M. Kopecky, A. Lausi, R.H. Menk, M. Miculin, and A. Savoia, Phys. Rev. B 62, 5273 (2000).

${ }^{33}$ A. Erbil, G.S. Cargill, III, R. Frahm, and R.F. Boehme, Phys. Rev. B 37, 2450 (1998).

${ }^{34}$ W.T. Elam, J.P. Kirkland, R.A. Neiser, and P.D. Wolf, Phys. Rev. B 38, 26 (1988).

${ }^{35}$ M. Tegze, G. Faigel, S. Marchesini, M. Belakhovsky, and A.I. Chumakov, Phys. Rev. Lett. 82, 4847 (1999).

${ }^{36}$ T. Hiort, D.V. Novikov, E. Kossel, and G. Materlik, Phys. Rev. B 61, R830 (2000).

${ }^{37}$ S.S. Fanchenko, D.V. Novikov, A. Schley, and G. Materlik, Phys. Rev. B 66, 060104 (2002).

${ }^{38}$ J.J. Barton, Phys. Rev. Lett. 67, 3106 (1991).

${ }^{39}$ M. Tegze, G. Faigel, S. Marchesini, M. Belakhovsky, and O. U1rich, Nature (London) 407, 38 (2000).

${ }^{40}$ S. Marchesini and C.S. Fadley, Phys. Rev. B 67, 024115 (2003).

${ }^{41}$ F.N. Chukhovskii and A.M. Poliakov, Acta Crystallogr., Sect. A: Found. Crystallogr. 59, 109 (2003).

${ }^{42}$ P. Korecki, G. Materlik, and J. Korecki, Phys. Rev. Lett. 86, 1534 (2001).

${ }^{43}$ Y. Takahashi, K. Hayashi, and E. Matsubara, Phys. Rev. B 68, 052103 (2003).

${ }^{44}$ Y. Nishino and G. Materlik, Phys. Rev. B 60, 15074 (1999).

${ }^{45}$ Y. Nishino, L. Troger, P. Korecki, and G. Materlik, Phys. Rev. B 64, 201101 (2001).

${ }^{46}$ Y. Nishino, T. Ishikawa, M. Suzuki, N. Kawamura, P. Kappen, P. Korecki, N. Haack, and G. Materlik, Phys. Rev. B 66, 113103 (2002).

${ }^{47}$ M. Kopecky, A. Lausi, E. Busetto, J. Kub, and A. Savoia, Phys. Rev. Lett. 88, 185503 (2002).

${ }^{48}$ M. Tegze, G. Faigel, and S. Marchesini, Phys. Rev. Lett. 89, 279601 (2002).

${ }^{49}$ M. Kopecky, A. Lausi, E. Busetto, J. Kub, and A. Savoia, Phys. Rev. Lett. 89, 279602 (2002).

${ }^{50}$ S.G. Bompadre, T.W. Petersen, and L.B. Sorensen, Phys. Rev. Lett. 83, 2741 (1999).

${ }^{51}$ P. Korecki and G. Materlik, Phys. Rev. Lett. 86, 2333 (2001). 